\title{
La ramification des extensions galoisiennes est déterminée par les discriminants de certaines sous-extensions
}

\author{
par
}

FranÇOIS LaUbie (Limoges)

Introduction. Soit $p$ un nombre premier. Dans cet article, on désigne par corps local un corps complet pour une valuation discrète dont le corps résiduel est parfait de caractéristique $p$. Etant données deux extensions galoisiennes de corps locaux $L / K$ et $L^{\prime} / K^{\prime}$ dont les groupes de Galois $G$ et $G^{\prime}$ sont isomorphes, on dit qu'un isomorphisme de groupes $\varrho$ de $G$ sur $G^{\prime}$ est un isomorphisme de ramification de $G$ sur $G^{\prime}$ ou de $L / K$ sur $L^{\prime} / K^{\prime}$ s'il respecte les filtrations de ramification de $G$ et de $G^{\prime}$, c'est-à-dire si pour tout $u \in\left[0, \infty\left[\right.\right.$, on a $\varrho\left(G^{u}\right)=G^{\prime u}$; dans le cas où $L / K$ et $L^{\prime} / K^{\prime}$ sont des extensions finies, il est équivalent d'imposer que pour tout $i \in \mathbb{N}$, on a $\varrho\left(G_{i}\right)=G_{i}^{\prime}$. S'il existe un isomorphisme de ramification de $L / K$ sur $L^{\prime} / K^{\prime}$, on dit que ces extensions sont identiquement ramifiées.

Un isomorphisme de groupes $\varrho$ de $G$ sur $G^{\prime}$ induit par la théorie de Galois une bijection croissante entre les ensembles ordonnés par l'inclusion des sousextensions $E / K$ de $L / K$ et $E^{\prime} / K^{\prime}$ de $L^{\prime} / K^{\prime}$; sauf en cas d'ambigüité cette bijection sera encore notée $\varrho$.

L'objet de cet article est de déterminer des ensembles $\mathcal{E}$ de sous-extensions finies de $L / K$ vérifiant la propriété suivante : tout isomorphisme de groupes $\varrho$ de $G$ sur $G^{\prime}$ tel que, pour tout $E / K \in \mathcal{E}$, les valuations des discriminants des extensions $E / K$ et $E^{\prime} / K^{\prime}=\varrho(E / K)$ coïncident, est un isomorphisme de ramification de $L / K$ sur $L^{\prime} / K^{\prime}$. Nous montrons que dans le cas le plus général où $L / K$ est une extension galoisienne finie ou infinie, l'ensemble de toutes les sous-extensions finies de $L / K$ convient et que dans le cas où $L / K$ est une extension galoisienne finie on peut prendre pour $\mathcal{E}$ l'ensemble des sous-extensions de $L / K$ qui sont invariantes par les sousgroupes cycliques de $G$; nous montrons également que dans le cas particulier où $K$ et $K^{\prime}$ sont des corps locaux d'inégales caractéristiques et où $L / K$ est une extension de Lie $p$-adique [7], il existe un tel ensemble $\mathcal{E}$ qui est fini; nous montrons enfin que dans le cas où $K$ et $K^{\prime}$ sont des extensions finies de $\mathbb{Q}_{p}$ de même indice de ramification $e$ et où $L / K$ est une extension abélienne dont 
la sous-extension non ramifiée maximale $L_{0} / K$ est finie, on peut prendre pour $\mathcal{E}$ l'ensemble des sous-extensions finies de $L / K$ dont les valuations des discriminants ne dépassent pas $\left(q^{v}-1\right)^{2} /(q-1)$ où $q$ désigne le nombre d'éléments du corps résiduel de $L_{0}$ et où $v=1+[e p /(p-1)]$.

Comme exemple d'applications, ces résultats interviennent pour décider quelles sont les extensions de corps locaux qui sont identiquement ramifiées à des extensions de corps locaux à corps résiduel fini [2].

Isomorphismes de ramification. Soient $L / K$ et $L^{\prime} / K^{\prime}$ deux extensions galoisiennes de corps locaux dont les groupes de Galois $G$ et $G^{\prime}$ sont isomorphes et soit $\varrho$ un isomorphisme de groupes de $G$ sur $G^{\prime}$.

Soit $u_{L / K}$ (resp. $u_{L^{\prime} / K^{\prime}}$ ) la fonction d'ordre de la filtration de ramification de $G$ (resp. de $G^{\prime}$ ) en numérotation supérieure :

$$
u_{L / K}(\sigma)=u \Leftrightarrow \forall \varepsilon>0, \sigma \in G^{u}-G^{u+\varepsilon} .
$$

Pour que $\varrho$ soit un isomorphisme de ramification il faut et il suffit que $u_{L / K}=u_{L^{\prime} / K^{\prime}} \circ \varrho$.

Dans le cas où $L / K$ est une extension finie ou arithmétiquement profinie au sens de Fontaine et Wintenberger [1], c'est-à-dire quand les groupes de ramification sont des sous-groupes ouverts du groupe de Galois, la filtration de ramification de $G$ admet également une numérotation inférieure $i_{L / K}=\psi_{L / K} \circ u_{L / K}$ où la fonction $\psi_{L / K}$ de Hasse-Herbrand est définie par $\psi_{L / K}(x)=\int_{0}^{x}\left(G^{0}: G^{u}\right) d u([5]$, ch. IV, $\S 3)$. Si $L / K$ et $L^{\prime} / K^{\prime}$ sont des extensions finies ou arithmétiquement profinies, pour que $\varrho$ soit un isomorphisme de ramification il faut et il suffit que $i_{L / K}=i_{L^{\prime} / K^{\prime}} \circ \varrho$.

Soit $E / K$ une sous-extension de $L / K$ et soit $E^{\prime} / K^{\prime}=\varrho(E / K)$; si $E / K$ est galoisienne, il en est de même de $E^{\prime} / K^{\prime}$ et $\varrho$ induit un isomorphisme de groupes $\bar{\varrho} \operatorname{de} \operatorname{Gal}(E / K) \operatorname{sur} \operatorname{Gal}\left(E^{\prime} / K^{\prime}\right)$.

Proposition 1. Si @ est un isomorphisme de ramification de $L / K$ sur $L^{\prime} / K^{\prime}$ alors $\varrho$ est un isomorphisme de ramification de $E / K$ sur $E^{\prime} / K^{\prime}$.

Démonstration. C'est une conséquence immédiate du théorème de Herbrand ([5], ch. IV, §3) qui fonctionne aussi bien pour les extensions infinies que pour les extensions finies.

Soit $J$ un ensemble ordonné filtrant à droite et soit $\left\{E_{j}\right\}_{j \in K}$ une famille de sous-corps de $L$ contenant $K$ telle que $\forall i, j \in I, i \leq j \Rightarrow E_{i} \subset E_{j}$ et $L=\bigcup_{j \in J} E_{j}$. On suppose que toutes les extensions $E_{j} / K$ sont galoisiennes; il en est alors de même des extensions $E_{j}^{\prime} / K^{\prime}=\varrho\left(E_{j} / K\right)$ et pour tout $j \in J$, on note $\bar{\varrho}_{j}$ l'isomorphisme de $\operatorname{Gal}\left(E_{j} / K\right)$ sur $\operatorname{Gal}\left(E_{j}^{\prime} / K^{\prime}\right)$ induit par $\varrho$.

Proposition 2. Si pour tout $j \in J$, $\bar{\varrho}_{j}$ est un isomorphisme de ramification de $E_{j} / K$ sur $E_{j}^{\prime} / K^{\prime}$ alors $\varrho$ est un isomorphisme de ramification de $L / K \operatorname{sur} L^{\prime} / K^{\prime}$. 
Démonstration. Pour tout $j \in J$, soit $H_{j}=\operatorname{Gal}\left(L / E_{j}\right)$ et $H_{j}^{\prime}=$ $\operatorname{Gal}\left(L^{\prime} / E_{j}^{\prime}\right)=\varrho\left(H_{j}\right)$. Dans l'identification de $G$ (resp. de $G^{\prime}$ ) avec la limite projective canonique de $G / H_{j}$ (resp. des $G^{\prime} / H_{j}^{\prime}$ ), on a d'après le théorème de Herbrand, $G^{u}=\lim G^{u} H_{j} / H_{j}=\lim _{\longleftarrow}\left(G / H_{j}\right)^{u}$ et $G^{u}=\lim \left(G^{\prime} / H_{j}^{\prime}\right)^{u}$ pour tout $u \geq 0$; donc $G^{\prime u}=\lim _{\longleftarrow} \bar{\varrho}_{j}\left(\left(G / H_{j}\right)^{u}\right)=\varrho\left(\lim _{\longleftarrow}\left(G / H_{j}\right)^{u}\right)=\varrho\left(G^{u}\right)$.

Proposition 3. Soit $E / K$ une sous-extension finie de $L / K$ et soit $E^{\prime} / K^{\prime}=\varrho(E / K)$. Si @ est un isomorphisme de ramification de $L / K$ sur $L^{\prime} / K^{\prime}$, alors sa restriction à $\mathrm{Gal}(L / E)$ est un isomorphisme de ramification de $L / E$ sur $L^{\prime} / E^{\prime}$.

Démonstration. Soit $J$ un ensemble ordonné filtrant à droite et $\left\{E_{j}\right\}_{j \in J}$ une famille d'extensions galoisiennes finies de $K$ contenues dans $L$ contenant $E$, telle que $\bigcup_{j \in J} E_{j}=L$ et $\forall j_{1}, j_{2} \in J, j_{1} \leq j_{2} \Rightarrow E_{j_{1}} \subset$ $E_{j_{2}}$; on note $E_{j}^{\prime} / K^{\prime}=\varrho\left(E_{j} / K\right)$ et $\bar{\varrho}_{j}$ l'isomorphisme de $\operatorname{Gal}\left(E_{j} / K\right)$ sur $\operatorname{Gal}\left(E_{j}^{\prime} / K^{\prime}\right)$ induit par $\varrho$ qui, d'après la proposition 1, est un isomorphisme de ramification. Comme, pour tout entier naturel $i$, le groupe de ramification d'indice $i$ (en numérotation inférieure) de l'extension $E_{j} / E$ est la trace sur $\operatorname{Gal}\left(E_{j} / E\right)$ du groupe de ramification d'indice $i$ de l'extension $E_{j} / K$ ([5], ch. IV, §1), la restriction $\bar{\varrho}_{j}^{\prime}$ de $\bar{\varrho}_{j}$ à $\operatorname{Gal}\left(E_{j} / E\right)$ est un isomorphisme de ramification de $E_{j} / E$ sur $E_{j}^{\prime} / E^{\prime}$. Il résulte alors de la proposition 2 que la restriction de $\varrho$ à $\operatorname{Gal}(L / E)$, qui s'identifie à la limite projective des $\varrho_{j}^{\prime}$, est un isomorphisme de ramification.

Remarques. 1. Dans [2], il y a une autre preuve de la proposition 3 valable seulement dans le cas d'extensions finies.

2. Supposons que $\varrho$ soit un isomorphisme de groupes de $G$ sur $G^{\prime}$ qui induise canoniquement des isomorphismes de ramification d'une sous-extension galoisienne $E / K$ de $L / K$ sur son image $E^{\prime} / K^{\prime}=\varrho(E / K)$ et aussi de $L / E$ sur $L^{\prime} / E^{\prime}$; alors même si les extensions $L / K$ et $L^{\prime} / K^{\prime}$ sont identiquement ramifiées, $\varrho$ n'est pas nécessairement un isomorphisme de ramification.

Contre-exemple. Soient $K=\mathbb{Q}_{2}, L=K(\sqrt{-1}, \sqrt{2}), E=K(\sqrt{-2})$ $\subset L, H=\operatorname{Gal}(L / E)$ et soit $\varrho$ l'automorphisme de $G$ qui échange les sousgroupes $\operatorname{Gal}\left(L / \mathbb{Q}_{2}(\sqrt{-1})\right)$ et $\operatorname{Gal}\left(L / \mathbb{Q}_{2}(\sqrt{2})\right)$ en laissant $H$ invariant; alors $\varrho$ induit l'identité sur $H$ et sur $G / H$ mais ce n'est pas un isomorphisme de ramification puisque $G_{0}=G_{1}=G, G_{2}=G_{3}=\operatorname{Gal}(L / K(\sqrt{-1}))$ et $G_{4}=(1)$.

Proposition 4. Supposons que $L / K$ et $L^{\prime} / K^{\prime}$ soient des extensions finies ou arithmétiquement profinies. Soit $H$ un groupe de ramification de $L / K$ et soit $E$ le corps de ses invariants. Si l'isomorphisme @ de $G$ sur $G^{\prime}$ induit canoniquement des isomorphismes de ramification de $E / K$ sur $E^{\prime} / K^{\prime}=\varrho(E / K)$ et de $L / E$ sur $L^{\prime} / E^{\prime}$, alors $\varrho$ est lui-même un isomorphisme de ramification. 
Démonstration. Soit $i_{0} \in \mathbb{N}$ tel que $H=G_{i_{0}}$ et notons $\varrho(H)=H^{\prime}$. Montrons d'abord que $H^{\prime}=G_{i_{0}}^{\prime}$. On a d'une part $H_{i_{0}}^{\prime}=H^{\prime} \cap G_{i_{0}}^{\prime}$ et d'autre part $H_{i_{0}}^{\prime}=\varrho\left(H_{i_{0}}\right)=H^{\prime}$ donc $G_{i_{0}}^{\prime} \supset H^{\prime}$. Soit $\psi^{\prime}$ la fonction $\psi$ de HasseHerbrand associée à l'extension de groupe de Galois $H^{\prime}$ (loc.cit.); comme $H_{i_{0}}^{\prime}=H^{\prime}$ on a $\psi^{\prime}\left(i_{0}\right)=i_{0}$. Soit $\psi^{\prime \prime}$ la fonction $\psi$ de Hasse-Herbrand associée à l'extension de groupe de Galois $G_{i_{0}}^{\prime} / H^{\prime}$ et soit $u_{0}$ tel que $\psi^{\prime \prime}\left(u_{0}\right)=i_{0}$; alors, d'après le théorème de Herbrand, on a

$$
\left(G_{i_{0}}^{\prime} / H^{\prime}\right)_{i_{0}}=\left(G_{i_{0}}^{\prime} / H^{\prime}\right)^{u_{0}}=\left(G_{i_{0}}^{\prime}\right)^{u_{0}} / H^{\prime}=\left(G_{i_{0}}^{\prime} \cap G_{\psi^{\prime}\left(i_{0}\right)}^{\prime}\right) / H^{\prime}=G_{i_{0}}^{\prime} / H^{\prime} ;
$$

mais par hypothèses on a aussi

$$
\left(G_{i_{0}}^{\prime} / H^{\prime}\right)_{i_{0}} \subset\left(G^{\prime} / H^{\prime}\right)_{i_{0}}=(\bar{\varrho}(G / H))_{i_{0}}=\bar{\varrho}\left((G / H)_{i_{0}}\right)=\bar{\varrho}((1))=(1) ;
$$

donc $G_{i_{0}}^{\prime}=H^{\prime}$.

Soit maintenant un entier naturel $i$. Si $i \geq i_{0}$ alors $G_{i} \subset H$ et $\varrho\left(G_{i}\right)=$ $\varrho\left(H_{i}\right)=H_{i}^{\prime}=G_{i}^{\prime} \cap H^{\prime}=G_{i}^{\prime}$. Si $i \leq i_{0}$ alors $G_{i} \supset H$ et ([5], ch. IV, $\S 1$, cor. à la prop. 3) on a $\varrho\left(G_{i}\right) / H^{\prime}=\varrho\left(G_{i} / H\right)=\varrho\left((G / H)_{i}\right)=\left(G^{\prime} / H^{\prime}\right)_{i}=G_{i}^{\prime} / H^{\prime}$, ce qui signifie que $\varrho\left(G_{i}\right)=G_{i}^{\prime}$.

Proposition 5. Soient $K$ et $K^{\prime}$ deux extensions finies de $\mathbb{Q}_{p}$ de même indice de ramification e. Soient $L / K$ et $L^{\prime} / K^{\prime}$ deux extensions abéliennes de groupes de Galois $G$ et $G^{\prime}$ isomorphes. On pose $\alpha=e /(p-1)+1$ et $\beta=\alpha+e$ et on suppose que $L / K$ est totalement ramifiée. Soit $\varrho$ un isomorphisme de groupe de $G$ sur $G^{\prime}$. Si @ induit par passage au quotient un isomorphisme de ramification $\bar{\varrho}$ de $G / G^{\beta}$ sur $G^{\prime} / \varrho\left(G^{\beta}\right)$ alors $\varrho$ est un isomorphisme de ramification.

Démonstration. Pour tout groupe $J$ on note $P(J)$ l'ensemble des puissances $p$-èmes des éléments de $J$. D'après la théorie du corps de classe local, pour tout $n \geq \alpha$ on a $P\left(G^{n}\right)=G^{n+e}$ et en particulier $P\left(G^{\alpha}\right)=G^{\beta}$ ([5], ch. XIV, $\S 4$, prop. 9); de plus les sauts de ramifications supérieurs de $G$ sont entiers ([5], ch. IV, $\S 3$, th. de Hasse-Arf). Soit $H=G^{\beta}$ et $H^{\prime}=\varrho\left(G^{\beta}\right)$; d'après le théorème de Herbrand, on a

$$
\varrho\left(H G^{n}\right) / H^{\prime}=\bar{\varrho}\left((G / H)^{n}\right)=\left(G^{\prime} / H^{\prime}\right)^{n}=G^{\prime n} H^{\prime} / H^{\prime},
$$

donc pour tout $n \in \mathbb{N}, H^{\prime} \varrho\left(G^{n}\right)=H^{\prime} G^{\prime n}$; en particulier pour $n \leq \beta$, $\varrho\left(G^{n}\right)=H^{\prime} G^{\prime n}$ et il s'ensuit que

$$
\begin{aligned}
H^{\prime} & =\varrho(H)=\varrho\left(P\left(G^{\alpha}\right)\right)=P\left(\varrho\left(G^{\alpha}\right)\right) \\
& =P\left(H^{\prime} G^{\prime \alpha}\right)=P\left(H^{\prime}\right) P\left(G^{\prime \alpha}\right)=P\left(H^{\prime}\right) G^{\prime \beta} \\
& =P\left(P\left(H^{\prime}\right) G^{\prime \beta}\right) G^{\prime \beta}=P^{2}\left(H^{\prime}\right) G^{\prime \beta}=\ldots=P^{m}\left(H^{\prime}\right) G^{\prime \beta}
\end{aligned}
$$

pour tout entier $m \geq 1$. Mais $H^{\prime}$ est un pro-p-groupe, donc l'ensemble des $P^{m}\left(H^{\prime}\right)$ est un système fondamental de voisinages de l'élément neutre dans $H^{\prime}$ et aussi dans $G^{\prime}$ puisque $H^{\prime}$ est ouvert dans $G^{\prime}$ et comme $G^{\prime \beta}$ est fermé, 
on a $H^{\prime}=\bigcap_{m \geq 1}\left(P^{m}\left(H^{\prime}\right) G^{\prime \beta}\right)=G^{\prime \beta}$. Il en résulte que pour tout $n \leq \beta$, $\varrho\left(G^{n}\right)=G^{\prime n}$.

La preuve s'achève par récurrence sur $n_{0}$ tel que pour tout $n \leq n_{0}$ on ait $\varrho\left(G^{n}\right)=G^{\prime n}$; en effet, pour tout $n \in\left\{n_{0}+1, n_{0}+2, \ldots, n_{0}+e\right\}$, on a

$$
\varrho\left(G^{n}\right)=\varrho\left(P\left(G^{n-e}\right)\right)=P\left(\varrho\left(G^{n-e}\right)\right)=P\left(G^{\prime n-e}\right)=G^{\prime n} .
$$

Discriminants et ramification. Outre les notations précédentes qui restent valides, étant donné un corps local $K$, on note encore $v_{K}$ la valuation discrète de $K$ normalisée par $v_{K}\left(K^{*}\right)=\mathbb{Z}$ et $\bar{K}$ le corps résiduel de $K$. De plus, si $L$ est une extension finie de $K$ les symboles $e(L / K), f(L / K), D_{L / K}$ et $d(L / K)$ désignent respectivement l'indice de ramification de $L / K$, le degré résiduel de $L / K$, le discriminant de $L / K$ et la valuation $v_{K}\left(D_{L / K}\right)$ du discriminant de $L / K$; autrement dit, $D_{L / K}=\left\{x \in K: v_{K}(x) \geq d(L / K)\right\}$.

Enfin, si $H$ est un groupe d'automorphismes de $L, L^{H}$ désigne comme d'habitude le corps des invariants de $H$.

ThÉorème. Soient $L / K$ et $L^{\prime} / K^{\prime}$ deux extensions galoisiennes finies de corps locaux dont les groupes de Galois $G$ et $G^{\prime}$ sont isomorphes et soit $\varrho$ un isomorphisme de groupes de $G$ sur $G^{\prime}$. On suppose que pour tout sous-groupe cyclique $H$ de $G$ les discriminants des extensions $L^{H} / K$ et $L^{\prime \varrho(H)} / K^{\prime}$ ont même valuation. Alors @ est un isomorphisme de ramification.

Démo nstration. Montrons d'abord que, sous les hypothèses ci-dessus, $\varrho$ applique le groupe d'inertie $G_{0}$ de $L / K$ sur le groupe d'inertie $G_{0}^{\prime}$ de $L^{\prime} / K^{\prime}$. Soient $\sigma \in G, \sigma^{\prime}=\varrho(\sigma), H$ le sous-groupe de $G$ engendré par $\sigma, H^{\prime}=\varrho(H), E=L^{H}$ et $E^{\prime}=L^{\prime H^{\prime}}$. Pour que $\sigma \in G_{0}$ (resp. $\left.\sigma^{\prime} \in G_{0}^{\prime}\right)$ il faut et il suffit que toute sous-extension $F / E$ de $L / E$ (resp. $F^{\prime} / E^{\prime}$ de $\left.L^{\prime} / E^{\prime}\right)$ soit ramifiée, c'est-à-dire que $d(F / E)=0\left(\right.$ resp. $\left.d\left(F^{\prime} / E^{\prime}\right)=0\right)$ ou encore, compte tenu de la formule de transitivité des discriminants ([5], ch. III, §4), que $d(F / K)>d(E / K)$ (resp. $\left.d\left(F^{\prime} / K^{\prime}\right)>d\left(E^{\prime} / K^{\prime}\right)\right)$.

Or comme $L / F$ et $L / E$ sont des extensions cycliques, ces dernières inégalités sont respectées simultanément.

Maintenant, comme le groupe de ramification $G_{1}$ (resp. $G_{1}^{\prime}$ ) est le $p$ sous-groupe de Sylow de $G_{0}$ (resp. de $G_{0}^{\prime}$ ) il est clair que $\varrho$ applique $G_{1}$ sur $G_{1}^{\prime}$. Soit $L_{1}$ (resp. $L_{1}^{\prime}$ ) le corps des invariants de $G_{1}$ (resp. de $G_{1}^{\prime}$ ). Soit $E / L_{1}$ une sous-extension de $L / L_{1}$ telle que $\operatorname{Gal}(L / E)$ soit cyclique et soit $E^{\prime} / L_{1}^{\prime}=\varrho\left(E / L_{1}\right)$. La formule de transitivité des discriminants (loc. cit.) s'écrit $d(E / K)=\left[E: L_{1}\right] d\left(L_{1} / K\right)+f(L / K) d\left(E / L_{1}\right)$ ou $d\left(E^{\prime} / K^{\prime}\right)=$ $\left[E^{\prime}: L_{1}^{\prime}\right] d\left(L_{1}^{\prime} / K^{\prime}\right)+f(L / K) d\left(E^{\prime} / L_{1}^{\prime}\right)$; on a donc $d\left(E^{\prime} / L_{1}^{\prime}\right)=d\left(E / L_{1}\right)$. On a donc prouvé qu'il suffit de démontrer le théorème lorsque $L / K$ est une extension galoisienne finie totalement sauvagement ramifiée et que, dans ce cas, l'extension $L^{\prime} / K^{\prime}$ est également totalement sauvagement ramifiée. 
Supposons donc que $L / K$ et $L^{\prime} / K^{\prime}$ sont totalement sauvagement ramifiées. On rappelle que dans ces conditions si $m$ est un entier non multiple de $p$, pour tout $\sigma \in G$, on a $i_{L / K}\left(\sigma^{m}\right)=i_{L / K}(\sigma)$ parce qu'en désignant par $\pi$ une uniformisante de $L$, si $\sigma(\pi) \equiv \pi\left(1+a \pi^{i}\right) \bmod \pi^{i+2}$ alors $\sigma^{m}(\pi) \equiv$ $\pi\left(1+\operatorname{ma}^{i}\right) \bmod \pi^{i+2}$. Soit $\theta$ l'application de $G$ dans $\mathbb{Z}$ définie par $\theta(\sigma)=$ $i_{L / K}(\sigma)-i_{L^{\prime} / K^{\prime}}(\varrho(\sigma))$ si $\sigma \neq 1$ et $\theta(1)=0$.

Montrons que $\theta=0$. Soit $E / K$ une sous-extension de $L / K$ telle que le groupe de Galois $H$ de $L / E$ soit un sous-groupe cyclique de $G$. Soit $E^{\prime} / K^{\prime}=\varrho(E / K), H^{\prime}=\varrho(H)$ et soit $\mathcal{D}$ (resp. $\left.\mathcal{D}^{\prime}\right)$ la différente de l'extension $L / E$ (resp. $\left.L^{\prime} / E^{\prime}\right)$. On a alors

$$
\begin{aligned}
v_{L}(\mathcal{D}) & =d(L / E)=d(L / K)-[L: E] d(E / K) \\
& =d\left(L^{\prime} / K^{\prime}\right)-\left[L^{\prime}: E^{\prime}\right] d\left(E^{\prime} / K^{\prime}\right)=d\left(L^{\prime} / E^{\prime}\right)=v_{L^{\prime}}\left(\mathcal{D}^{\prime}\right),
\end{aligned}
$$

donc

ou encore

$$
\sum_{\sigma \in H, \sigma \neq 1}\left(1+i_{L / K}(\sigma)\right)=\sum_{\sigma^{\prime} \in H^{\prime}, \sigma^{\prime} \neq 1}\left(1+i_{L^{\prime} / K^{\prime}}\left(\sigma^{\prime}\right)\right),
$$

$$
\sum_{\sigma \in H} \theta(\sigma)=0
$$

Nous pouvons maintenant démontrer par récurrence sur l'ordre de $G$ que $\theta=0$. Soit $\sigma$ un élément de $G$ d'ordre $\nu>1$ et soit $H$ le sousgroupe cyclique engendré par $\sigma$. Soit $\tau \in H$; si $\tau$ est un générateur de $H$ alors il existe un entier $m$ premier à $p$ tel que $\tau=\sigma^{m}$, donc $i_{L / K}(\tau)=$ $i_{L / K}(\sigma), i_{L^{\prime} / K^{\prime}}(\varrho(\tau))=i_{L^{\prime} / K^{\prime}}(\varrho(\sigma))$ et $\theta(\tau)=\theta(\sigma)$; si $\tau$ engendre un sousgroupe cyclique de $G$ strictement inclu dans $H$, en appliquant l'hypothèse de récurrence à la restriction de $\theta$ à ce sous-groupe on obtient $\theta(\tau)=0$. Il en résulte que $0=\sum_{\tau \in H} \theta(\tau)=\varphi(\nu) \theta(\sigma)$ où $\varphi(\nu)$ est l'indicateur d'Euler de $\nu$, et donc que $\theta(\sigma)=0$.

Pour tout $\sigma \in G$ on a donc $i_{L^{\prime} / K^{\prime}}(\varrho(\sigma))=i_{L / K}(\sigma)$ et $\varrho$ est bien un isomorphisme de ramification.

Remarques. 1. Ce théorème admet clairement un énoncé analogue avec les différentes à la place des discriminants.

2. La preuve de ce théorème publiée dans [2] dans le cas d'extensions totalement ramifiées est incomplète car la représentation d'Artin n'est pas toujours rationnelle sur $\mathbb{Q}$ (voir [6], $\S 13$ et plus spécialement le théorème $30^{\prime}$ ).

Corollaire 1. Soient $L / K$ et $L^{\prime} / K^{\prime}$ deux extensions galoisiennes (infinies) de corps locaux dont les groupes de Galois $G$ et $G^{\prime}$ sont isomorphes et soit $\varrho$ un isomorphisme de groupes de $G$ sur $G^{\prime}$. On suppose que pour toute sous-extension galoisienne finie $E / K$ de $L / K$ les discriminants de $E / K$ et de $\varrho(E / K)$ ont même valuation. Alors @ est un isomorphisme de ramification. 
Démonstration. Il résulte du théorème que pour tout sous-groupe ouvert distingué $H$ de $G, \varrho$ induit par passage au quotient un isomorphisme $\bar{\varrho}$ de $G / H$ sur $G^{\prime} / \varrho(H)$ qui est un isomorphisme de ramification de $L^{H} / K$ sur $L^{\prime \varrho(H)} / K^{\prime}$ et la conclusion découle alors immédiatement de la proposition 2.

Rappelons que les extensions de Lie de corps locaux sont les extensions galoisiennes dont les groupes de Galois sont des groupes de Lie p-adiques et dont les groupes d'inertie sont des sous-groupes d'indices finis des groupes de Galois [7]. Les extensions de Lie sont arithmétiquement profinies ([4], [7]) et, en inégales caractéristiques, leurs ramifications jouissent de remarquables propriétés de régularité [4].

Corollaire 2. Soient $K$ et $K^{\prime}$ deux corps locaux de caractéristique nulle et de même indice de ramification absolu. Soient $L / K$ et $L^{\prime} / K^{\prime}$ deux extensions de Lie dont les groupes de Galois $G$ et $G^{\prime}$ sont isomorphes. Il existe alors un entier naturel $d_{0}$ vérifiant la propriété suivante: tout isomorphisme de groupe @ de $G$ sur $G^{\prime}$ qui, parmi les valuations des discriminants de sous-extensions finies de $L / K$, conserve celles qui ne dépassent pas $d_{0}$, est un isomorphisme de ramification.

Démonstration. Soit $L_{0} / K$ la sous-extension non ramifiée maximale de $L / K$ et soit $e$ l'indice de ramification absolu de $L_{0}$. On sait ([3], prop. 4) qu'il existe un nombre réel $w$ vérifiant la propriété suivante : pour tout corps local $F^{\prime}$ d'indice de ramification absolu $e$, pour toute extension galoisienne $L^{\prime}$ de $F^{\prime}$ de groupe de Galois $H^{\prime}$ isomorphe à $G^{0}$, si un isomorphisme de groupes $\varrho$ de $G^{0}$ sur $H^{\prime}$ induit un isomorphisme de ramification de $G^{0} / G^{w}$ sur $H^{\prime} / \varrho\left(G^{w}\right)$, alors c'est un isomorphisme de ramification. Soit $M$ le corps des invariants de $G^{w}$ et soit $d_{0}=d(M / K)$ la valuation du discriminant de $M / K$. Supposons que $\varrho$ conserve les valuations des discriminants des sous-extensions $E / K$ de $M / K$, c'est-à-dire que $d\left(E^{\prime} / K^{\prime}\right)=d(E / K)$ avec $E^{\prime} / K^{\prime}=\varrho(E / K)$; alors, d'après le théorème, $\varrho$ induit par passage au quotient un isomorphisme de ramification de $M / K$ sur $M^{\prime} / K^{\prime}=\varrho(M / K)$ et aussi de $M / L_{0}$ sur $M^{\prime} / F^{\prime}$ où $F^{\prime} / K^{\prime}$ désigne la sous-extension non ramifiée maximale de $M^{\prime} / K^{\prime}$. Il en résulte que la restriction de $\varrho$ à $G_{0}$ est un isomorphisme de ramification de $L / L_{0}$ sur $L^{\prime} / F^{\prime}$; en particulier, $F^{\prime} / K^{\prime}$ est la sous-extension non ramifiée maximale de $L^{\prime} / K^{\prime}$ et donc $\varrho$ est un isomorphisme de ramification.

R e marque. Il serait intéressant de savoir si l'on peut choisir pour $d_{0}$ un entier ne dépendant que du groupe d'inertie $G_{0}$ de $L / K$ et du corps de ses invariants $L_{0}$. Lorsque l'extension de Lie $L / K$ est abélienne, on peut choisir pour $d_{0}$ un entier ne dépendant que de $L_{0}$.

Corollaire 3. Soient $K$ et $K^{\prime}$ deux extensions finies de $\mathbb{Q}_{p}$ de même indice de ramification e. Soient $L / K$ et $L^{\prime} / K^{\prime}$ deux extensions abéliennes 
dont les groupes de Galois $G$ et $G^{\prime}$ sont isomorphes. On suppose que la sous-extension non ramifiée maximale $L_{0} / K$ de $L / K$ est finie. Soit q le nombre d'éléments du corps résiduel de $L_{0}$, soit

$$
v=\left[\frac{e p}{p-1}\right]+1
$$

et soit

$$
d_{0}=\frac{\left(q^{v}-1\right)^{2}}{q-1} .
$$

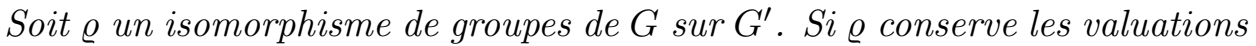
des discriminants des sous-extensions de $L / K$ qui sont inférieures à $d_{0}$ alors @ est un isomorphisme de ramification.

Dém on stration. D'après la proposition 5 , le nombre réel $w$ introduit dans la démonstration du corollaire 2 peut ici être choisi égal à $[e p /(p-1)]+$ $1=v$. Soit $M$ le corps des invariants de $G^{v}$. Par la même démonstration que celle du corollaire 2 , si on a $d\left(E^{\prime} / K^{\prime}\right)=d(E / K)$ pour toutes sous-extensions $E / K$ de $M / K$ avec $E^{\prime} / K^{\prime}=\varrho(E / K)$, alors $\varrho$ est un isomorphisme de ramification. Il suffit donc de montrer que $d(M / K) \leq d_{0}$.

Or on a $d(M / K)=\sum_{\sigma \in G^{0} / G^{v}, \sigma \neq 1}(1+i(\sigma))$ avec $i=i_{L / K}$. Soit $\psi$ la fonction de Hasse-Herbrand associée à $M / K$ et soit $u(\sigma)=\sup \{u \in \mathbb{R}: \sigma \in$ $\left.G^{u} / G^{v}\right\}$. On a

$$
\begin{aligned}
i(\sigma) & =\psi(u(\sigma))=\int_{0}^{u(\sigma)}\left(G^{0} / G^{v}: G^{t} / G^{v}\right) d t=\int_{0}^{u(\sigma)}\left(G^{0}: G^{t}\right) d t \\
& =\sum_{j=1}^{u(\sigma)} \int_{j-1}^{j}\left(G^{0}: G^{t}\right) d t \leq q+q^{2}+\ldots+q^{u(\sigma)}
\end{aligned}
$$

parce que $\left(G^{x}: G^{x+1}\right) \leq q([5]$, ch. IV, $\S 2)$ et si $x \notin \mathbb{N},\left(G^{x}: G^{x+1}\right)=1$ (théorème de Hasse-Arf). On a donc

$$
\begin{aligned}
d(M / K) & \leq \sum_{\sigma \in G^{0} / G^{v}, \sigma \neq 1}\left(1+q+q^{2}+\ldots+q^{u(\sigma)}\right) \\
& \leq\left(\left(G^{0}: G^{v}\right)-1\right)\left(1+q+q^{2}+\ldots+q^{v-1}\right)=\frac{\left(q^{v}-1\right)^{2}}{q-1}=d_{0} .
\end{aligned}
$$

Exemple. Soient $L$ et $L^{\prime}$ deux extensions abéliennes de $\mathbb{Q}$ de groupes de Galois isomorphes $G$ et $G^{\prime}$. Soit $\varrho$ un isomorphisme de $G$ sur $G^{\prime}$. Si pour tout corps de nombres $E \subset L$ dont la $p$-partie du discriminant est $\leq p^{(p-1)(p+1)^{2}}(p \neq 2)$, les $p$-parties des discriminants de $E$ et de $\varrho(E)$ coïncident, alors $p$ se ramifie identiquement dans $L$ et dans $L^{\prime}$. 


\section{Bibliographie}

[1] J.-M. Fontaine et J.-P. Wintenberger, Le "corps des normes" de certaines extensions algébriques de corps locaux, C. R. Acad. Sci. Paris Sér. A 288 (1979), 367-370.

[2] F. Laubie, Groupes de ramification et corps résiduels, Bull. Sci. Math. (2) 105 (1981), 309-320.

[3] - Sur la ramification des extensions de Lie, Compositio Math. 55 (1985), 253-262.

[4] S. Sen, Ramification in p-adic Lie extensions, Invent. Math. 17 (1972), 44-50.

[5] J.-P. Serre, Corps locaux, 2-ème éd., Hermann, Paris, 1968.

[6] -, Représentations linéaires des groupes finis, 2-ème éd., Hermann, Paris, 1971.

[7] J.-P. Wintenberger, Extensions de Lie et groupes d'automorphismes des corps locaux de caractéristique p, C. R. Acad. Sci. Paris Sér. A 288 (1979), 477-479.

[8] - Le corps des normes de certaines extensions infinies de corps locaux; applications, Ann. Sci. École Norm. Sup. (4) 16 (1983), 59-89.

DÉPARTEMENT DE MATHÉMATIQUES

FACULTÉ DES SCIENCES

123, AV. A. THOMAS

87060 LIMOGES CEDEX, FRANCE 